\title{
La logique institutionnelle d'appropriation du concept Thinkpad University : le cas des écoles et des facultés de gestion québécoises francophones (1996-2010)
}

Patrick Pelletier

TÉLUQ-Université du Québec

\section{Résumé}

L'objectif de cet article est de favoriser une meilleure compréhension de la manière dont se construisent les processus de changement institutionnel en contexte d'enseignement supérieur. Nous le faisons par l'étude de la logique institutionnelle d'appropriation du concept Thinkpad University de l'entreprise IBM que l'on retrouve dans le champ institutionnel des écoles et des facultés de gestion québécoises. Notre démarche inspirée du néo-institutionnalisme sociologique s'appuie sur un modèle constitué de trois plans d'analyse : institutionnel, organisationnel et opérationnel. Nous relevons, d'une part, la variabilité avec laquelle les organisations se conforment aux pressions institutionnelles et, d'autre part, les processus qui favorisent l'institutionnalisation de pratiques technopédagogiques. S'avèrent déterminants dans ces processus, des facteurs tels le prestige institutionnel, la mission organisationnelle, l'autonomie décisionnelle et l'expertise pédagogique. Nous soutenons que la source de la logique institutionnelle d'appropriation relève d'une quête de réputation.

\begin{abstract}
This study examines the process by which a change in institutional logic of a organizational field is initiated. More specifically, we are concerned with how the IBM Thinkpad University concept affects various business schools in the
\end{abstract}


province of Quebec. The theoretical framework departs from new-institutional theories. We propose a multi-level analysis: institutional, organizational and operational. The finding examines the process by which organizations retain, adopt and discard practices and illustrates the influence of institutional prestige, organizational mission, autonomy and pedagogical expertise. We show that the source of the institutional logic of appropriation is a quest for reputation.

L'objectif de cet article est de favoriser une meilleure compréhension de la manière dont se construisent les processus de changement institutionnel en contexte d'enseignement supérieur. Nous portons notre attention sur la manière dont des idées sont retenues en tant que solutions positives dans les organisations évoluant en ce contexte. Nous le faisons par l'étude de la logique institutionnelle (Thornton \& Occasio, 2008) d'appropriation du concept Thinkpad University de l'entreprise IBM dans les écoles et facultés de gestion québécoises francophones. Le concept renvoie à la présence d'un environnement d'apprentissage où il y a des projecteurs et des prises de connexion internet dans les classes, les amphithéâtres et les espaces publics, ainsi qu'un intranet et des services de soutien technopédagogique et d'assistance technique. Alors que les étudiants sont tenus de faire l'achat d'un ordinateur portatif standardisé et configuré selon les logiciels nécessaires à leur apprentissage, les professeurs sont invités de leur côté à exploiter les possibilités de l'environnement institué en développant des pratiques technopédagogiques en classe, à distance ou encore en mode hybride. Comment se construit la logique institutionnelle d'appropriation du concept Thinkpad University? Quelles sont les répercussions de cette logique sur les pratiques qui caractérisent traditionnellement les écoles et facultés de gestion québécoises francophones? Et que nous apprennent cette logique et ces répercussions à propos du processus d'institutionnalisation des technopédagogies en contexte d'enseignement supérieur? Tel sont les questionnements qui guident la réflexion dont il est question dans le présent article, réflexion qui a pour ancrage théorique le néoinstitutionnalisme sociologique (Dimaggio \& Powell, 1983, 1991).

Le néo-institutionnalisme sociologique est une approche sociocognitiviste s'intéressant aux cadres d'action collective influençant les processus de construction sociale (Berger \& Luckmann, 1967) des pratiques que développent les organisations. Les forces qui mènent ces dernières au changement ne relèvent pas pour cette approche que de l'efficacité et de la rationalité : il y a dans tout environnement des croyances portées par des institutions qui établissent des critères par lesquels les organisations découvrent leurs préférences. On défend alors l'idée que ce n'est pas tant l'efficacité qui est recherchée par ces dernières, mais la légitimité auprès des acteurs qui leur sont externes et qui ont un pouvoir de sanction à leur égard.

Bien que des études relevant du néo-institutionnalisme sociologique aient été menées dans le contexte de l'enseignement supérieur, il n'existe pas d'écrits à notre connaissance qui traitent précisément du processus de changement institutionnel lié à des pratiques technopédagogiques. Qui plus est, les analyses ont porté sur des pratiques institutionnalisées et non sur les processus qui mènent ou non à leur institutionnalisation, c'est-à-dire du passage de leur statut d'innovation à celui de pratique habituelle, 
constamment répétée, voire considérée comme normale. En d'autres termes, une pratique est institutionnalisée lorsqu'elle est tenue pour acquise puisque jugée légitime (Tolbert \& Zucker, 1996; Greenwood, Suddaby \& Hinings, 2002). Dans les écrits scientifiques, on retrouve notamment, comme objets d'étude, la professionnalisation des programmes de formation (Kraatz \& Zajac, 1996), les accréditations des écoles et facultés de gestion (Casile \& Davis-Blake, 2002) ainsi que la variation des processus d'embauche dans la gestion de programmes de recyclage (Lounsbury, 2001). Ces études ont en commun d'être axées sur le pouvoir d'influence des institutions, laissant peu de place aux organisations qui, par une certaine réflexivité, construisent les pratiques. Il en est de même d'autres recherches qui touchent à différents contextes organisés (Frenkel, 2005; Carney \& Farashasi, 2005; Salmeron \& Bueno, 2006; Boiral, 2006; Hopper \& Major, 2007; Ezzamel \& al., 2007).

La démarche proposée ici va de pair avec les nouvelles orientations du néoinstitutionnalisme sociologique qui s’intéressent davantage aux dimensions individuelles (Dacin, Goodstein \& Scott, 2002; Greenwood \& Suddaby, 2006; Delbridge \& Edwards, 2008; Battilana, Leca \& Boxenbaum, 2009; Battilana \& D'aunno, 2009) et organisationnelles (Goodrick \& Salancik, 1996; Townley, 2002; Danisman, Hinings \& Slack, 2006; Battalina \& D’Aunno, 2009) liées aux processus de changement institutionnel. Pour ce faire, l'intentionnalité stratégique et la réflexivité que manifestent les acteurs font l'objet d'une plus grande attention dans les analyses (Quinn \& Washington, 2009; Zietsma \& McNight, 2009; Battalina \& D’Aunno, 2009). Le chercheur est amené à considérer davantage la présence d'acteurs ayant une intention d'initier un changement divergent quant aux normes institutionnelles qui encadrent traditionnellement leurs actions. Ces acteurs peuvent créer, entre autres, des occasions de changement en tirant profit des contradictions présentes dans l'environnement dans lequel ils évoluent.

Le choix de s'intéresser au concept Thinkpad dans une perspective néo-institutionnelle n'est pas sans fondement puisque, contrairement aux pratiques qui ont fait l'objet d'études jusqu'à présent, son appropriation ne relève pas de facto de normes institutionnelles, que ce soit celles définies par l'État ou encore celles d'associations professionnelles. Le développement des pratiques technopédagogiques liées à la logique institutionnelle à l'étude relève plutôt du bon vouloir de différents acteurs, particulièrement des professeurs. Il s'agit d'acteurs dont la pratique sur le plan pédagogique n'est pas traditionnellement formalisée, mais plutôt artisanale. Bien qu'elles bénéficient d'un fort appui au plan institutionnel depuis de nombreuses années, les pratiques technopédagogiques, que ces acteurs développent, coexistent avec d'autres pratiques perçues comme étant davantage centrales à la réalisation de la mission des organisations dans lesquelles ils évoluent : les activités de recherche et d'enseignement traditionnel en classe. Parce qu'elles s'avèrent alors périphériques, mais aussi innovantes, et qu'elles prennent forme dans un environnement jugé des plus soumis aux pressions institutionnelles (Dimaggio \& Powell, 1983; Meyer \& Rowan, 1977) et où le changement relève d'un processus lent et intuitif qui se construit par plusieurs modifications successives plus ou moins restreintes sur une période relativement longue (Mintzberg, 2004), ces pratiques nous paraissent des plus intéressantes à la compréhension de ce que sont les processus de changement institutionnel. Des processus complexes qui, selon Agre (2002), sont loin d'être neutres lorsqu'il est question d'intégration des TIC en contexte d'enseignement supérieur. Ce dernier avance que beaucoup d'innovations potentielles seraient impraticables sans 
qu'une masse critique de campus les utilise. Une fois qu'une masse critique d'utilisateurs est atteinte, les avantages d'opter pour une innovation sont nombreux. En raison de cette dynamique, les choix faits par les premières organisations peuvent s'avérer fatidiques pour celles qui suivent leur exemple. Par le fait qu'il porte une attention particulière aux processus d'influence qui ont cours entre les organisations en contexte de changement, le néo-institutionnalisme sociologique constitue une approche théorique des plus légitimes à notre démarche.

\section{Le cadre théorique}

Le néo-institutionnalisme sociologique poursuit trois principaux objectifs. Il est question de comprendre : 1) comment les choix sociaux qui supportent le développement des pratiques au sein des organisations sont formés et canalisés par des arrangements institutionnels; 2) comment s'exercent les pressions institutionnelles afin que les organisations s'y conforment et 3) comment ces pressions influencent les organisations alors que celles-ci les modifient et les sélectionnent. Ces pressions sont de nature réglementaire (les lois et les règlements soutenus par un système coercitif centralisé qui assure leur respect, l'État, par exemple), normative (les normes et les valeurs à atteindre et devant être respectées puisque assurées par des mécanismes sociaux propres aux participants, les associations professionnelles, entre autres) et cognitive (les représentations symboliques tenues pour acquises) (Scott, 2001).

Leconcept dechamp(Dimaggio \& Powell, 1983) permetdesituerlelieu où se construisent les pressions institutionnelles. Il se définit par l'agrégation d'organisations qui partagent un sentiment d'appartenance et qui interagissent autour d'un projet à construire ou d'une problématique à résoudre (Hoffman, 2001). Sa définition demeure par conséquent une activité subjective (Greenwood, Suddaby \& Hinings, 2002). Un champ est en soi un contexte qui, pour faire référence à Weick (1979), n'existe que dans les limites des perceptions des organisations et des acteurs qui les constituent. Tout acteur, qu'il soit individuel ou collectif, construit son environnement, autant qu'il est construit par lui au fil des interactions sociales.

Que ce soit par l'étude des processus coercitif (pressions sociales formelles et informelles exercées par les institutions et les attentes culturelles d'une société), normatif (origine dans la professionnalisation des groupes transversaux dont les pratiques font l'objet de conformité) ou mimétique (imitation par adoption des standards de référence liés à la performance face à l'incertitude), le néo-institutionnalisme sociologique nous apprend que les organisations se conforment au pouvoir des institutions pour augmenter leur chance de survie, au nom de la quête de la légitimité (Suchman, 1995). Or, bien qu'elle permette d'éviter l'ambiguïté, de faciliter l'acquisition de ressources auprès des parties prenantes et de résoudre les imperfections des informations dont les organisations disposent, l'obtention de cette légitimité n'est pas pour autant synonyme d'efficacité (Dimaggio \& Powell, 1983). Comme le souligne Rouleau (2007), c'est particulièrement le cas dans les organisations situées au sein d'environnements fortement institutionnalisés, tels que les établissements d'enseignement supérieur.

Afin de rendre compte de la manière dont se construisent les processus de changement dans le contexte institutionnel à l'étude, le présent article propose trois plans d'analyse : institutionnel (les processus d'influence qui amènent les organisations à agir dans un sens 
donné au nom de la légitimité), organisationnel (les processus décisionnels entourant le développement de l'expertise technopédagogique) et opérationnel (les processus de production des nouvelles pratiques et de reproduction des pratiques traditionnelles d'enseignement).

\section{Le cadre méthodologique}

$\mathrm{Du}$ paradigme phénoménologique duquel on peut distinguer l'approche interprétativiste et constructiviste, notre démarche repose sur la première approche, au sens que nous portons notre attention sur les perceptions des acteurs. Le cadre méthodologique utilisé se veut inductif modéré (Thietart, 1999) et nous privilégions des cas multiples et holistes (Yin, 1994). Six organisations ( $A, B, C, D, E$ et $F$ ) constituent notre échantillon. En raison du fait que les écoles et facultés de gestion québécoises recouvrent une pluralité d'organisations, notre étude est précisément axée sur les établissements francophones, lesquels se distinguent des établissements anglophones en tant que champ institutionnel. Il importe, ce faisant, de cerner le processus de changement institutionnel, lié à ces organisations dont l'action est encadrée dans la spécificité linguistique minoritaire canadienne, à l'heure de l'internationalisation de l'enseignement supérieur.

Le choix de considérer la période 1996-2010 s'explique par le fait que les premiers discours québécois associés à l'appropriation du concept Thinkpad sont apparus au début de cette période. Dans l'objectif de cerner les frontières du champ institutionnel, l'historicité des processus d'influence qui y ont cours a été retracée.

L'entretien de type semi-directif (Romelaer, 2005), mené auprès de différents répondants dans les six organisations qui constituent le champ à l'étude, s'est avéré la méthode de choix afin de faire ressortir des données nous permettant de saisir les spécificités du processus de changement institutionnel. Un guide d'entretien, formalisé à partir de deux entretiens exploratoires menés dans les organisations $A$ et $B$, a servi d'aide-mémoire. Les entretiens (d'une durée de 45 à 120 minutes) débutaient par des questions d'ordre individuel : formation, parcours, fonction, expertises pédagogique et technopédagogique des répondants. Puis, les points suivants liés au développement des pratiques étaient abordés : éléments déclencheurs, objectifs, influences, facteurs facilitants, contraintes, points tournants et répercussions.

Vingt-deux entretiens semi-directifs ont été menés (d'octobre 2006 à février 2010) auprès de dix-huit répondants qui se différencient selon deux catégories : les acteurs administratifs et les acteurs pédagogiques. Quatre acteurs de $A$ et $B$ ont fait l'objet d'un deuxième entretien (après un an et demi) afin de prendre connaissance des transformations survenues au cours de la période à l'étude. Les acteurs ont été choisis en fonction du rôle décisif qu'ils ont joué quant à l'appropriation $(A$ et $B)$ ou non $(C, D, E$ et $F)$ du concept Thinkpad. Huit acteurs administratifs (doyens, vice-doyens, directeurs généraux et directeurs de programme) ont été rencontrés dans les six organisations. Dix acteurs pédagogiques (professeurs innovateurs (7), professeur chargé de projet (1) et conseillers technopédagogiques (2) ont été rencontrés dans $A$ (6) et $B$ (4). Les professeurs ont été choisis en fonction du fait que le développement de leurs pratiques technopédagogiques a reçu une certaine visibilité, notamment pour l'obtention d'un prix.

Les entretiens ont été complétés par diverses sources documentaires : des énoncés de principes (5) démontrant la légitimité des projets; des procès-verbaux de réunions 
(4) entourant leur planification et mise en œuvre; des sites internet (6) relatant des informations à propos des pratiques développées; une étude de cas (1); un article scientifique (1) à propos d'une pratique et des articles de journaux (3). Utiliser ces différentes méthodes et ressources (Lincoln \& Guba, 1985) permettait de réaliser l'objectif de retenir les justifications (pourquoi?) et les manières (comment?) autour de la logique institutionnelle d'appropriation du concept Thinkpad. Cette triangulation a permis de répondre en partie à la difficulté de reconstruire son historicité. Les discours des acteurs et des textes ont été retranscrits, lus et relus, codifiés et re-codifiés (Corbin \& Strauss, 1990). Un premier codage a permis de relever les concepts émergents. Une répétition des codages nous a conduits à délimiter un ensemble de catégories d'analyse.

\section{Présentation et analyse des résultats}

Sur le plan institutionnel, le processus de changement relève de l'habileté des acteurs à développer une argumentation légitime auprès des acteurs dominants du champ institutionnel, ce qui en soi, n'est pas différent d'autres contextes (Edelman \& Suchman, 1997). En s'appuyant sur l'exemple de pratiques reconnues à l'échelle internationale et de l'intérêt d'acteurs institutionnels voulant se faire partenaires, l'organisation $A$ construit une réalité par une nouvelle rationalité technique qui permet la création de nouvelles occasions. $B$ s'appuie quant à elle sur l'exemple de $A$. Ces organisations se comparent en fait avec celles qui correspondent à leurs espoirs et attentes quant à l'avenir. Or, la différence avec sa propre situation devient une définition du problème à résoudre pour reprendre les termes de Sahlin-Anderson (1996), pour qui le changement repose sur des stratégies de problématisation. La logique institutionnelle à l'œuvre ne renvoie pas tant à la volonté de résoudre un problème vécu, mais plutôt de répondre à une occasion qui paraît légitime en raison des bénéfices économiques qui lui sont associés. La source de cette logique institutionnelle est une quête de réputation. Les acteurs mobilisent de ce fait la rationalité concurrentielle qu'incarne le marché avant tout autre type de rationalité. Il s'ensuit différents effets d'entraînement entre les organisations. Celles qui adoptent le concept le font par un processus de création identitaire (Czarniawska \& Wolff, 1998) présentant une vision de l'avenir. Nous constaterons que la logique institutionnelle ne traduit pas pour autant une transformation du champ, mais plutôt l'accentuation de la différenciation qui le caractérise déjà. Cette dernière est liée à des facteurs tels le prestige institutionnel, la mission organisationnelle (élitisme versus démocratisation/capital économique des étudiants), l'autonomie décisionnelle (école versus faculté) et l'expertise pédagogique acquise au cours des années. Les répercussions de la logique sont marquées des modes de compétition (positionnement stratégique) et de collaboration (transfert des connaissances).

Sur le plan organisationnel, les organisations développent des stratégies d'implantation qui auront des répercussions différenciées sur l'expertise technopédagogique. Cette dernière n'est pas indépendante de la culture spécifique de l'organisation et du rapport à l'innovation qui y prévaut. Sur le plan opérationnel, les technopédagogies constituent une avenue exploratoire qui donne lieu à différentes expérimentations et innovations. 


\section{Le plan institutionnel : les processus d'influence}

Le début de la période à l'étude est associé à la pression que représente l'intégration des NTICà l'échelleinternationale. Y répondre devient une priorité pour la direction de $A$. Alors que celle-ci met l'emphase sur la présence d'une nouvelle génération d'étudiants rompue à l'utilisation de ces technologies, et sur l'importance d'un nouveau contexte marqué de la réingénierie des processus dans les entreprises, l'acteur qui sera éventuellement choisi pour effectuer l'implantation du concept Thinkpad soulève a posteriori quelques problèmes organisationnels liés à un environnement d'apprentissage qui n'était pas approprié : surcharge des locaux informatiques et non-standardisation des logiciels, particulièrement pour l'enseignement des sciences comptables. Le contexte s'avérait des plus favorables pour le développement d'un environnement technopédagogique intégré dans $A$, puisqu'il était déjà prévu que cette organisation bénéficierait dans un avenir rapproché d'un nouvel édifice.

C'est au début de l'année 1995 que convaincre le Ministre de l'Éducation de doter $A$ des infrastructures technologiques nécessaires s'impose. Il s'agit d'un ministre perçu à l'époque comme étant un fervent défenseur de l'intégration des NTIC dans tous les ordres d'enseignement au Québec. Dans un document produit par des acteurs de $A$, il est précisé qu'une soumission aux tendances internationales concernant l'intégration des NTIC « se traduirait par la perte de ce qui est un joyau parmi les actifs de formation en gestion du Québec et du Canada. Cela priverait également le Québec d'un moteur potentiel d'affirmation dans l'économie virtuelle en émergence ». Il est question de devenir une université virtuelle d'envergure mondiale qui devra " contrer ses concurrents qui, grâce à la flexibilité de leurs systèmes de livraison, pourront dans un proche avenir offrir à la clientèle des produits de formation plus performants à un coût compétitif ». On souligne que les relations avec les entreprises québécoises et les firmes du monde entier se resserreront considérablement du fait des communications électroniques sans failles. On présente la perspective future d'un espace régional formé de petites et moyennes entreprises québécoises, mais aussi de certains États américains. Le perfectionnement professionnel destiné à certaines industries est visé. À la question de desservir des marchés éloignés, on s'adonne à un exercice de comparaison avec différents programmes de formation en ligne les plus reconnus à l'échelle internationale. On souligne que de nombreuses grandes entreprises en télécommunication se feront partenaires. Devant un argumentaire marqué du sceau de l'innovation pédagogique et du positionnement stratégique pour le Québec, l'accord ministériel aura lieu à l'été 1995.

Les mois passent et le projet défendu jusqu'alors ne prendra pas forme pourtant, puisqu'il n'a jamais été question du concept Thinkpad. La direction de $A$, qui est jugée de nature entrepreneuriale par les acteurs qui y évoluent, réalise à la même époque qu'être la première organisation à se doter du concept Thinkpad au Québec pourrait constituer une stratégie de positionnement des plus enviables. Cela se traduit dans les termes suivants :

Parce qu'en 1994-1995, ils avaient commencé à lancer les premières universités Thinkpad aux États-Unis, entre autres au Minnesota [1993]. Donc, c'est [le directeur] qui a dit à un moment donné : « Il faut faire le virage. Nouvelle école... Il faut se positionner. 
Et pour le directeur du M.B.A., il demeurait important que son programme « soit bien positionné » (Acteur administratif 2, $A$ ).

Guidée à l'échelle nord-américaine par la promotion de son programme Global Campus Initiative, l'entreprise IBM entreprend à l'été 1996 la visite des écoles et facultés de gestion québécoises. Elle est désormais munie d'un argument de taille : $A$ est fort intéressée à devenir une université Thinkpad. En se rappelant les propos du représentant d'IBM à sa première visite dans $B$, un acteur administratif nous précisera qu'il :

[...] ne m'a pas convaincu. Il a dit que c'est un projet que l'on pourrait faire. Il avait dit que l'on en entendrait parler à $[A]$. [...] On ne nous présentait pas quelque chose de bien structuré. Le projet était de dire que les étudiants ont des ordinateurs portatifs et que cela vous évite dans le fond de vous équiper de laboratoires (Acteur administratif $1, B$ ).

À ses arguments s'en ajoute un autre qui est loin d'être négligeable : devenir une université Thinkpad est le plus souvent associé à une augmentation des inscriptions des étudiants. Or, cette organisation connaît depuis près de dix ans une décroissance sur le plan de ses effectifs. Celui qui sera chargé de mettre en place le nouvel environnement d'apprentissage dans $B$ affirme à propos d'un établissement américain:

L'histoire du Minnesota, elle est assez impressionnante parce qu'il y avait une université qui avait trois pavillons, deux au sud et un complètement au nord, et la direction avait évalué la fermeture de l'un d'entre eux. Bien, le département qui devait fermer, il est arrivé avec un projet comme cela. L'année où ils ont implanté, ils ont eu une augmentation de leurs inscriptions de $40 \%$... je pense (Acteur administratif $2, B$ ).

Mais encore fallait-il que $B$ puisse bénéficier des infrastructures nécessaires pour entreprendre le virage techno-pédagogique souhaité. Contrairement à $A$, l'organisation $B$ ne possède pas de nouvelle demeure. Seul le recteur pourrait convaincre les instances gouvernementales de doter l'organisation d'un nouvel environnement. Le projet consiste à faire des sciences de la gestion une vitrine de positionnement pour l'université d'attache. Le feu vert sera donné ultérieurement par le gouvernement tandis que l'association des étudiants et le milieu des entreprises de la région participeront au financement du projet. $A$ et $B$, les deux organisations les plus prestigieuses du champ à l'étude, pourront désormais rivaliser sur la base d'un environnement moderne sur le plan pédagogique et architectural.

Devenir une université Thinkpad s'avère porteur, mais encore fallait-il sonder l'opinion publique, vendre le concept. Les journaux serviront de vitrine. $A$ deviendra « la première université francophone virtuelle au monde ». La création d'une brochure promotionnelle jouera notamment un rôle clé, une tâche jugée difficile par ailleurs, car « comment annoncer de façon convaincante un programme dont les paramètres opérationnels n’avaient pas encore été définis! » (Étude de cas, 2005, $A$ ). De son côté, $B$ deviendra « la faculté de gestion du $3^{\mathrm{e}}$ millénaire ». Si le calendrier s'avère exigeant, $A$ n'a pas le loisir de retarder la mise en œuvre de son programme Thinkpad, de crainte de perdre l'avantage concurrentiel qu'elle recherche. Si $B$ ne peut bénéficier de la position du premier arrivé, elle sait qu'elle devra se différencier de $A$ en misant davantage sur l'expertise technopédagogique. 
Nous constatons que les acteurs de $C$ ne sont pas restés indifférents aux stratégies de $A$ et $B$, mais " C'est qu'on voyait mal comment... que les gens dépensent $3000 \$^{1}$ », nous dira-t-on avant d'ajouter que « la différence avec $[A]$ c'est qu'ils sont beaucoup plus libres que nous pouvons l'être. Il y a une combinaison de bureaucratie universitaire. Et j'aurais jamais été capable de défendre cela » (Acteur administratif $1, C$ ).

Largement influencée par $B$, qui a attiré une partie de son potentiel d'effectif d'étudiants dès la première année d'implantation du programme ThinkPad au baccalauréat, $D$ lance son programme deux ans plus tard. Devant le peu d'inscriptions, en raison notamment du capital économique des étudiants qui est inférieur à celui de $A$ et $B$, elle abandonne. Que dire de $E$ ? Son désintérêt est lié au fait que la spécificité pédagogique de son programme d'enseignement au baccalauréat s'avère alors une compétence distinctive lui servant d'avantage stratégique. Quant à $F$, elle décidera d'investir dans un programme d'apprentissage en ligne à distance pour répondre aux particularités de ses étudiants de deuxième cycle. Il n'est pas question du concept Thinkpad mais plutôt d'une réponse à celui-ci. Ainsi :

Il faut que l'on en fasse. Toutes les universités en font, il va falloir en faire. Puis, par ce que nous sommes sur multicampus... et que $[B]$ qui est notre concurrent local avait carrément décidé d’investir beaucoup dans la technologie comme façon de se démarquer. On ne pouvait pas avoir l'air arriéré technologiquement (Acteur administratif, $F$ ).

« Cela fait partie de la modernité institutionnelle », témoignera un autre acteur administratif de la même organisation.

L'appropriation du concept ThinkPad dans $A$ et $B$ mènera à une hausse des effectifs étudiants dès les premières années, qui s'atténuera dans les années ultérieures. Comme l'affirmera l'acteur administratif 1 de $B$ à la fin de l'année 2006 :

La conclusion du point de vue de l'étudiant c'est qu'évidemment aujourd'hui c'est de l'acquis. [...] On le fait encore pour le branding... mais maintenant ça fait partie des meubles. [...] La technologie est toujours là mais on est passé à autre chose.

Il s'agit ici d'un constat lié à l'image de marque et aux effets de mode qui est d'ailleurs aussi présent dans $A$. L'offre de programmes d'échanges internationaux aux étudiants constitue la voie privilégiée de positionnement pour $A$ et $B$ depuis 2005. Bien que $C, D, E$ et $F$ n'aient pas adopté le concept ThinkPad, quelques années plus tard, elles ont dû investir sur le plan technologique dans les classes. Comme le souligne l'acteur administratif 1 de $C:$ : Pour être concurrentiel, il faut être équipé. Les jeunes vont dire : « êtes-vous équipés dans les classes ? Ça prend cela».

Pour terminer, notons que $A$ et $B$ joueront un rôle sur le plan du transfert des connaissances en technopédagogie au Québec, que ce soit par la création d'une chaire de recherche ou la participation à différents colloques. Un transfert qui se traduit par ailleurs par des journées d'étude organisées annuellement et des activités du réseau des Thinkpad Universities dont $A$ et $B$ seront les hôtes au Québec. On s'attarde aux questions d'infrastructure informatique, de financement, de soutien et de développement de matériel technopédagogique. 


\section{Le plan organisationnel : le développement de l'expertise technopédagogique}

Les questions d'accessibilité et de coût à supporter par les étudiants seront mises à l'avant-plan lors des négociations menant à l'appropriation du concept Thinkpad dans $A$ et $B$. Mais pour la direction de $A$, les entreprises sont prêtes à payer pour recruter des étudiants qui maîtriseraient les NTIC. Un programme de prêt sera mis sur pied. Il en sera de même pour $B$ dont le profil socio-économique des étudiants diffère de ceux de $A$. Les deux organisations se questionnent sur le nombre de cours où l'ordinateur portable sera utilisé en classe et la proportion d'enseignants qui voudront investir du temps pour acquérir les compétences technologiques nécessaires et pour développer des pratiques jugées innovantes.

Conscientes de la complexité du changement à construire, les directions de $A$ et $B$ adoptent des stratégies différentes. Dans $A$, on distribue à l'ensemble des professeurs un ordinateur portatif. On favorise une stratégie de soutien que l'on peut qualifier de volontariste : "Ce n'est pas un hasard, parce que moi je voulais que l'innovation vienne de la base ", souligne l'acteur administratif 1 . Le développement sera piloté par un professeur intéressé depuis longtemps par les technologies en enseignement, et le soutien pédagogique sera assumé par quelques étudiants du baccalauréat. Ils constitueront une équipe de consultants, laquelle se professionnalisera au cours des années. Voici un témoignage recueilli au début de 2007 :

L'emphase était davantage mise sur le développement des habiletés et moins sur le côté de l'intégration à la pédagogie. C'est compliqué parce que l'on commence à s'y mettre. Il n'y avait pas de structures qui permettaient de raccrocher et c'était plus facile de mettre un côté technologique en disant que ça serait les étudiants avec les profs qui feraient les cahiers des charges ou les profs arriveraient avec leur cahier bien pensé (Acteur pédagogique 1, $A$ ).

L'approche volontariste préconisée par $A$ ne semble pas avoir faitl'unanimité : «L'autre chose a été de positionner l'ordinateur pour qu'il soit attrayant pour les étudiants mais pas rebutant pour les professeurs. Sans mentir, cela a été très difficile » (Acteur administratif $2, A$ ). Il y a eu chez certains d'entre eux « le syndrome d'une perte de contrôle ». On ajoute que « perdre le contrôle de l'attention, perdre le contrôle du contenu, de la discipline, la technologie fait cela. Elle répartit le pouvoir » (Acteur pédagogique 2, $A$ ). Le constat est similaire pour $B$. L'acteur administratif 1 de cette organisation nous révélera que :

Il y a eu des expérimentations vraiment géniales et qui perdurent mais au-delà de cela je ne pourrais pas dire que c'est là qu'il y a eu une grande révolution. Je te dirais que l'on travaille encore pour essayer de voir comment.

L'organisation $B$ développe de son côté une stratégie de soutien que nous qualifions de participative. Elle fait appel dès le départ à l'expérience d'un acteur qui était alors chargé de cours. Celui-ci affirmera que :

L’idée c'était d'apprendre de nos expériences professionnelles, l'expérience de certains collègues qui avaient expérimenté et on apprenait un peu sur le tas. On a engagé 
quelqu'un qui était en technologies éducatives, qui avait une maîtrise en éducation. C'était pour nous aider au niveau de la réflexion (Acteur administratif 2, B).

Contrairement à ceux que l'on retrouve dans $A$, les ordinateurs attribués aux professeurs de $B$ ne seront pas entièrement subventionnés par l'organisation, comme le souligne l'acteur administratif 1 de $B$ à propos du message envoyé aux professeurs :

Mais, 60 \%-40 \%; j’ai financé plus que toi. Donc, je peux te demander un petit peu. Je leur disais que ça ne sert pas uniquement pour l'enseignement mais pour la recherche. Vous avez des fonds de recherche. Le prix était intéressant. L’idée de leur donner un an d'avance était pour qu'ils comprennent ce qu'est l'appareil. Et après cela on a mis une équipe pour les appuyer.

Cet appui est au cœur de la culture organisationnelle souhaitée par $B$, puisque chaque cours de première année se verra attribuer un budget de développement. Les propos suivants s'avèrent des plus intéressants quant aux conséquences de cette stratégie :

Mais de dire que j'avais trouvé cela en 1997... Au début ce n'était pas cela mais à mesure que l'on avançait je m'apercevais que, finalement il y avait une transformation organisationnelle qui se faisait et d'où l'intérêt d'investir dans le soutien. Parce que les premières années, c'était beaucoup d'expérimentations. On avait des profs qui affichaient le PowerPoint mais ils écrivaient au tableau (Acteur administratif $1, B)$.

On organise annuellement dans les deux organisations des salons d'exposition des innovations technopédagogiques. Le but est de susciter l'intérêt à innover, à favoriser le déploiement de nouvelles pratiques. Notons que $A$ a influencé $B$ pour la création de ce type d'événement. Signalons aussi que, par son volet commercial et logistique, le concept Thinkpad rapproche les structures de fonctionnement des universités de celles des entreprises. Il y a eu transformation organisationnelle sur le plan du développement des compétences technologiques, de la veille technologique, de la vente et livraison d'appareils, du service après-vente (réparations et offre d'appareils de dépannage), de l'accès au réseau et de l'assistance technique en tout temps. Cette transformation pose la question des dépenses récurrentes en termes de modernisation technologique.

Pour terminer, précisons que les organisations $C, D, E$ et $F$ ont des structures de soutien beaucoup moins formalisées que $A$ et $B$. Cette différenciation soulève ainsi la question de la dépendance des ressources et démontre que le concept ThinkPad s'est avéré un catalyseur sur le plan de la réflexion pédagogique.

\section{Le plan opérationnel : la production et la reproduction des pratiques}

La présence des ordinateurs en classe créera, dès la fin des années 90, quelques commotions chez les étudiants, lesquelles se sont progressivement atténuées dans la mesure où leurs prix d'achat sont de plus en plus abordables (de 3000 \& en 1997, à 1000 \$ en 2004) et l'aspect nouveauté semble s'être dissipé. On constate qu'il y a eu amélioration des compétences technologiques des étudiants au départ et une augmentation significative des communications entre eux et les professeurs. 
Une analyse réalisée en 2005 par $A$ illustre que l'on a plus particulièrement encouragé l'utilisation de l'ordinateur dans les disciplines quantitatives. Le tableau, les acétates en plastique et les fichiers PowerPoint sont demeurés les outils pédagogiques les plus utilisés. L'utilisation de ces fichiers que les étudiants peuvent télécharger sur une plate-forme de gestion de cours mène, en 2007, à un autre constat sur la question du développement et de l'évaluation des apprentissages :

Il y en a qui disent qu'on a régressé. Peut-être que nous avons régressé un petit peu avec les PowerPoint et tout cela. Ça fait un enseignement statique, très structuré, moins d'interaction... Moi ce qui me chagrine beaucoup c'est de voir les étudiants à la veille des examens qui font juste passer à travers les PowerPoint (Acteur administratif $2, A$ ).

Par ailleurs, $A$ se définit comme étant probablement l'école qui utilisait le plus l'ordinateur pour les examens : "Beaucoup d'examens. Une infrastructure robuste, mais aussi un projet pédagogique qui menait jusqu'à l'évaluation ", précise l'acteur administratif 2 . Au-delà des mesures d'évaluation, il y a l'utilisation que font les étudiants de l'ordinateur en classe : "C'est que nous on a eu évidemment le gros problème des étudiants qui chattaient en classe... On a eu ça en 98 ... Et là toutes les universités ont ça maintenant » (Acteurs administratif 2, $A$ ).

Unerecension sur les sites internet nous amèneà constater que $B$ sembleavoir davantage innové que $A$. En 2000, B détient le plus grand nombre de pratiques technopédagogiques, soutenues par des professeurs perçus comme d'excellents pédagogues. Répondre à une situation jugée problématique (adéquation de la formation, coordination des cours, développement de meilleurs apprentissages et développement de la motivation des étudiants); profiter des opportunités technologiques offertes; réaliser son désir de créer et se différencier; développer un programme de recherche et commercialiser son innovation, sont autant de croyances qui ont amené ces professeurs à innover dans $A$ et $B$. Au chapitre des contraintes, on évoque dans ces deux organisations la peur d'innover, le scepticisme et le conservatisme des collègues ainsi que les politiques de promotion. On remarque que, au cours des années, les professeurs développent de moins en moins de pratiques technopédagogiques. Dans $B$, un témoignage recueilli en 2009 souligne que " cela a fonctionné pendant longtemps et cela fonctionne encore, sauf que là, le phénomène que l'on voit, c'est que la cour est pleine. J'entends des échos et mes conseillers me disent que c'est plus difficile de les convaincre qu'avant (Acteur administratif $1, B$ ).

La hausse des exigences liées à la publication scientifique serait une des causes évoquées dans $A$ et $B$. On constate néanmoins que, depuis 2005, des outils tels le blogue, le forum de discussion, la simulation, le wiki ou encore le télévoteur sont de plus en plus utilisés. Force est de constater que de nouvelles pratiques d'enseignement ont vu le jour, en raison de l'expertise développée progressivement par les équipes de soutien technopédagogique. L'intérêt depuis 2009 pour l'apprentissage en ligne hybride serait, entre autres, révélateur des nouvelles avenues empruntées. Se retrouvant uniquement dans $A$ et $B$, cette pratique marque le début d'un nouveau cycle pour le développement des technopédagogies dans les sciences de la gestion. 


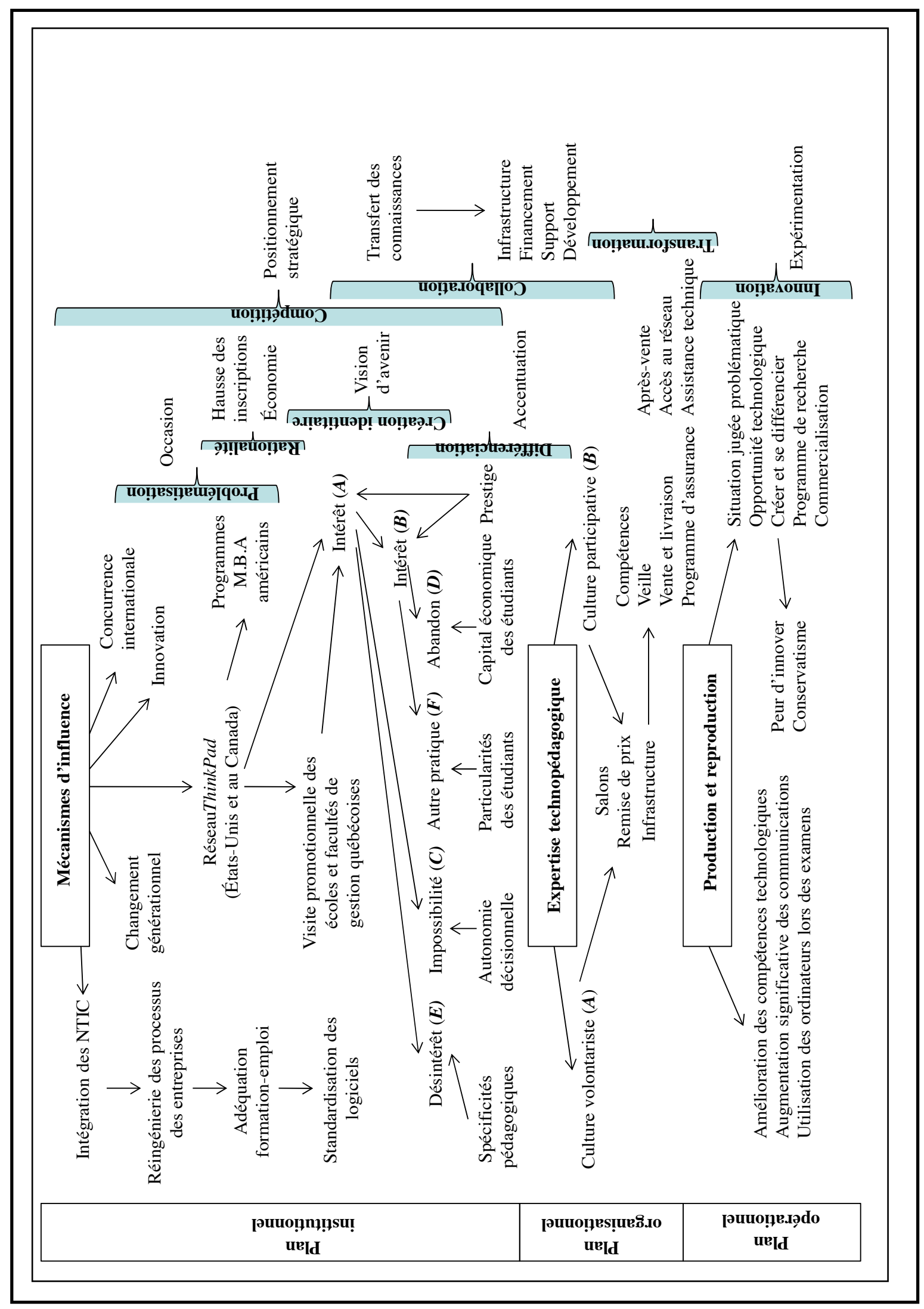




\section{Conclusion}

Par sa contribution empirique, le présent article révèle la manière dont peuvent se construire les processus de changement institutionnel en contexte d'enseignement supérieur, particulièrement en ce qui concerne les écoles et facultés de gestion québécoises francophones. Nous avons exposé les mécanismes d'influence, les effets de mode et les quêtes de réputation auxquels se prêtent ces organisations. Ces aspects révèlent, sur le plan sociologique, une des facettes symboliques de la modernité institutionnelle universitaire. La logique explicitée nous amène à dire que le processus de changement institutionnel relève d'une occasion. Les technopédagogies se présentent à la fin des années 90 et au début des années 2000 comme un puissant moteur de positionnement stratégique. La rationalité concurrentielle qu'incarne le marché est de fait mobilisée avant tout autre type d'explication d'institution. Puisque les attributs du concept Thinkpad ne sont pas conformes aux valeurs et aux normes qui caractérisent l'ensemble des organisations qui constituent le champ à l'étude, il y a création d'un "sous-champ » dont la figure institutionnelle de référence demeure les programmes M.B.A des «business schools » américaines. Au cours des années, ce sous-champ perd progressivement de son caractère de positionnement, si ce n'est de son attribut élitiste. En sont la cause des facteurs techniques telle la baisse du prix des ordinateurs personnels, mais aussi des facteurs culturels lorsque l'on évoque la présence d'étudiants intéressés à vivre des expériences à l'échelle internationale. La logique institutionnelle révèle par le fait même le caractère expérientiel de l'enseignement que recherchent ces étudiants. Cette logique à l'œuvre souligne aussi la manière avec laquelle les organisations recherchent la légitimité et répondent différemment aux pressions institutionnelles. Cette variabilité s'explique par l'influence de facteurs organisationnels : prestige institutionnel, mission organisationnelle, autonomie décisionnelle et expertise pédagogique. Notre étude comporte certaines limites, particulièrement en ce qui concerne l'évolution de l'appropriation des technopédagogies, de ses répercussions sur le plan des apprentissages et de ses utilisations différenciées selon les disciplines qui constituent les sciences administratives. Nous invitons les chercheurs à cerner d'autres logiques institutionnelles d'appropriation dans d'autres disciplines. Nous croyons fortement qu'il en appelle à une représentation plus exhaustive des tenants et aboutissants des technopédagogies à l'heure actuelle.

\section{Bibliographie}

Agre, P. E. (2002). Infrastructure and institutional change in the networked university. Dans W. H. Dutton \& B. D. Loader (dir.), Digital Academe; the New Media and Institutions of Higher Education and Learning, p. 152-166. London \& New York, NY : Routledge Taylor \& Francis Group.

Battilana, J. \& D'Aunno, T. (2009). Institutional work and the paradox of embedded agency. Dans T. B Lawrence, R. Suddaby \& B. Leca (dir.), Institutional work: Actors and Agency in Institutional Studies of Organization, p. 31-58. New York, NY : Cambridge University Press.

Battilana, J., Leca, B. et Boxenbaum, E. (2009). How actors change institutions; towards a theory of institutional entrepreneurship. The Academy of Management Annals, 3(1), 65-107. 
Berger, P. L. \& Luckmann, T. (1967). The social construction of reality: a treatise in the sociology of knowledge. New York, NY : Doubleday.

Boiral, O. (2006). La certification ISO 14001 : une perspective néo-institutionnelle. Management International, 10(3), 67-79.

Carney, M. \& Farashahi, G. (2006). Transnational institutions in developing countries: the case of iranian civil aviation. Organization Studies, 27(1), 53-77.

Casile, M. \& David-Blake, A. (2002). When accreditation standards change: factors affecting differential responsiveness of public and private organizations. Academy of Management Journal, 45(1), 180-195.

Corbin, J. \& Strauss, A. (1990). Grounded theory research - procedures, canons and evaluative criteria. Qualitative Sociology, 13(1), 3-21.

Czarniawska, B. \& Wolff, R. (1998). Constructing new identities in established organizational fields. International Studies of Management \& Organisation, 28(3), 32-56.

Dacin, M. T., Goodstein, J. \& Scott, W. R. (2002). Institutional theory and institutional change: introduction to the special research forum. Academy of Management Journal, 45(1), 450-57.

Danisman, A., Hinings, C. R. \& Slack, T. (2006). Integration and differenciation in institutional values: an empirical investigation in the field of canadian national sport organizations. Canadian Journal of Administrative Science / Revue canadienne des sciences de l'administration, 23(4), 301-317.

Delbridge, R. \& Edwards, T. (2008). Challenging conventions: roles and processes during non-isomorphic institutional change. Human Relations, 61(3), 299-325.

DiMaggio, P. J. \& Powell, W. W. (1983). The iron cage revisited: institutional isomorphism and collective rationality in organizational fields. American Sociological Review, 48(2), 147-160.

DiMaggio, P. J. \& Powell, W. W. (1991). The new institutionalism in organizational analysis. Chicago, IL : University of Chicago Press.

Edelman, L. B. \& Suchman, M. C. (1997). The legal environment of organizations. Annual Review of Sociology, 23, 479-515.

Ezzamel, M., Robson, K., Stapleton, P.\& Mclean, C. (2007). Discourse and institutional change: 'giving account' and accountability. Management Accounting Research, 18(2), $150-171$.

Frenkel, M. (2005). The politics of translation: how state-level political relations affect the cross-national travel of management ideas. Organization, 12(2), 275-301.

Goodrick, E. \& Salancik, G. R. (1996). Organizational discretion in responding to institutional practices: hospitals and cesarian births. Administrative Science Quarterly, 41(1), 1-28.

Greenwood, R., Suddaby, R. \& Hinings, C. R. (2002). Theorizing change: the role of professional associations in the transformation of institutionalized rules. Academy of Management Journal, 45(1), 58-80. 
Hoffman, A. J. (2001). Linking organizational and field-level analyses - the diffusion of corporate environmental practice. Organization \& Environnement, 14(2), 133-156.

Hopper, T. \& Major, M. (2007). Extending institutional analysis through theoretical triangulation: regulation and activity-based costing in portuguese telecommunications. European Accounting Review, 16(1), 59-97.

Kraatz, M. S. \& Zajac, E. J. (1996). Exploring the limits of the new institutionalism: the causes and consequences of illegitimate organizational change. American Sociological Review, 61(5), 812-836.

Lincoln, Y. \& Guba, E. (1995). Naturalistic Inquiry. Beverly Hills, CA : Sage.

Lounsbury, M. (2001). Institutional sources of practice variation: staffing college and university recycling program. Administrative Science Quarterly, 46(1), 29-56.

Meyer, J. \& Rowan, B. (1977). Institutionalized organizations: formal structure as myth and ceremony. American Journal of Sociology, 83(2), 340-363.

Mintzberg, H. (2004). Le management. Paris, France : Éditions d'Organisation.

Quinn, C. Q. \& Washington, M. (2009). Institutional work: actors and agency in institutional studies of organizations. Dans T. B, Lawrence, R. Suddaby \& B. Leca (dir.), Institutional work: actors and agency in institutional studies of organization, p. 236261. Londres, Royaume-Uni : Cambridge University Press.

Romelaer, P. (2005). L'entretien de recherche. Dans P. Roussel et F. Wacheux (dir.), Management des ressources humaines : méthodes de recherche en sciences humaines et sociales, p. 101-137. Bruxelles, Belgique : De Boeck.

Rouleau, L. (2007). Théories desorganisations; approchesclassiques, contemporaines et de l'avant-garde. Québec, Québec : Presses de l'Université du Québec.

Sahlin-Anderson, K. (1996). Imitating by editing success: the construction of organizational fields. Dans B. Czarniawska \& G. Sevón (dir.), Translating Organizational Change, p. 69-92. Berlin, Allemagne : de Gruyter.

Salmeron, J. L. \& Bueno, S. (2006). An information technologies and information systems industry-based classification in small and medium-sized enterprises: An institutional view. European Journal of Operational Research, 173, 1012-1025.

Scott, W. R. (2001). Institutions and organizations. Londres, Royaume-Uni : Sage Publications.

Suchman, M. C. (1995). Managing legitimacy: strategic and institutional approaches. Academy of Management Review, 20(3), 571-610.

Thietart, R. A. (1999). Méthodes de recherche en management. Paris, France : Dunod.

Thornton, P. H. \& Ocasio, W. (2008). Institutional logics. Dans Greenwood, R., Oliver, C. Sahlin, K. \& Suddaby, R. (Eds) Handbook of organizational institutionalism, p. 99129. Londres, Royaume-Uni : Sage.

Tolbert, P. S. \& Zucker, L. G. (1996). The institutionalization of institutional theory. Dans Clegg, C. R., Hardy, C. \& Nord, W. R. (dir.) Handbooks of organization studies, p. 175-190. Londres, Royaume-Uni : Sage. 
Townley, B. (2002). The role of competing rationalities in institutional change. Academy of Management, 45(1), 163-179.

Weick, K. (1979). The social psychology oforganizing. New York, NY : Random House.

Yin, R. K. (1994). Case study research - design and methods. Thousand Oaks, CA : Sage.

Zietsma, C. \& McNight, B. (2009). Building the iron cage: institutional creation work in the context of competing proto-institutions. Dans T. B Lawrence, R. Suddaby \& B. Leca (dir.). Institutional work: actors and agency in institutional studies of organization, p. 143-177. New York, NY : Cambridge University Press.

Zilber, T.B. (2006). The work of the symbolic in institutional processes: translations of rational myths in Israeli high tech. Academy of Management Journal, 49(2), 281-303.

\section{Note}

1. Montant que devait débourser un étudiant pour l'achat d'un ordinateur standardisé selon les exigences du programme.

\section{Coordonnées}

Patrick Pelletier

École des sciences de l'administration

TÉLUQ-Université du Québec

5800, rue Saint-Denis, Bureau 1105

Montréal, QC H2S 3L5 Canada

patrick.pelletier@teluq.ca

Patrick Pelletier est professeur de management à l'École des sciences de l'administration de la TÉLUQ-Université du Québec. Ses intérêts de recherche portent sur le changement en contexte d'enseignement supérieur. 